\title{
Observation of flat bands in twisted bilayer graphene
}

\author{
Simone Lisi ${ }^{1,9}$, Xiaobo Lu $\mathbb{1}^{2,9}$, Tjerk Benschop $\mathbb{1}^{3,9}$, Tobias A. de Jong ${ }^{3,9}$, Petr Stepanov $\mathbb{1}^{2}$, \\ Jose R. Duran², Florian Margot', Irène Cucchi', Edoardo Cappelli', Andrew Hunter', Anna Tamai ${ }^{1}{ }^{1}$, \\ Viktor Kandyba ${ }^{4}$, Alessio Giampietri ${ }^{4}$, Alexei Barinov ${ }^{4}{ }^{4}$, Johannes Jobst ${ }^{\mathbb{B}}{ }^{3}$, Vincent Stalman ${ }^{3}$, \\ Maarten Leeuwenhoek ${ }^{3,5}$, Kenji Watanabe ${ }^{1}{ }^{6}$, Takashi Taniguchi ${ }^{(10}{ }^{6}$, Louk Rademaker ${ }^{7}{ }^{7}$,

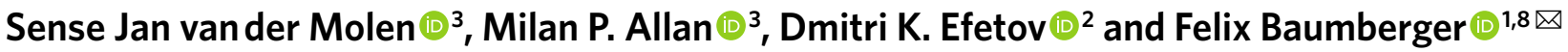

\begin{abstract}
Transport experiments in twisted bilayer graphene have revealed multiple superconducting domes separated by correlated insulating states $^{1-5}$. These properties are generally associated with strongly correlated states in a flat mini-band of the hexagonal moiré superlattice as was predicted by band structure calculations ${ }^{6-8}$. Evidence for the existence of a flat band comes from local tunnelling spectroscopy ${ }^{9-13}$ and electronic compressibility measurements ${ }^{14}$, which report two or more sharp peaks in the density of states that may be associated with closely spaced Van Hove singularities. However, direct momentum-resolved measurements have proved to be challenging ${ }^{15}$. Here, we combine different imaging techniques and angle-resolved photoemission with simultaneous real- and momentum-space resolution (nano-ARPES) to directly map the band dispersion in twisted bilayer graphene devices near charge neutrality. Our experiments reveal large areas with a homogeneous twist angle that support a flat band with a spectral weight that is highly localized in momentum space. The flat band is separated from the dispersive Dirac bands, which show multiple moiré hybridization gaps. These data establish the salient features of the twisted bilayer graphene band structure.

The small rotational misalignment of the sheets in twisted bilayer graphene (TBG) results in a long-range moiré superstructure with a unit cell that contains several thousand atoms. Moiré mini-bands, which lead to physical properties that deviate strongly from those of aligned bilayer graphene, can form in structurally highly perfect devices in which electronic states are coherent over multiple moiré unit cells and therefore over a great number of atomic sites. The formation of mini-bands further requires a finite overlap of the low-energy orbitals between neighbouring moiré sites, which in turn suggests the presence of extended wave functions with a weak on-site Coulomb repulsion. Nevertheless, near the magic twist angle of approximately $1.1^{\circ}$, TBG shows hallmarks of electron-electron correlations such as metal-insulator transitions, magnetism ${ }^{3,4}$, superconductivity $^{2,3,5}$ and departures from Fermi liquid behaviour in the metallic state ${ }^{16}$ that are more commonly observed in three-dimensional transition metal oxides with on-site interaction strengths of several $\mathrm{eV}$.
\end{abstract}

This dichotomy can be reconciled if there is a marked flattening of the dispersion in the moiré mini-bands, as predicted by band structure calculations. It is theoretically demanding to describe the electronic structure and related many-body physics of TBG given the size of its unit cell. Therefore, it is important to test key predictions of band structure calculations experimentally. However, this proved to be challenging, and direct electronic structure measurements by ARPES thus far have been largely limited to macroscopic samples of epitaxially grown bilayers with large and uncontrolled twist angles. Such measurements have shown signatures of the superlattice periodicity $^{17}$ and flat bands deep in the occupied states ${ }^{18}$, but have not shown evidence for the predicted partially filled flat band that is believed to be responsible for the correlated behaviour of TBG near the magic angle. Evidence for the latter has been reported in a very recent room-temperature study on a device made from exfoliated graphene that was strongly influenced by an additional moiré superlattice that arose from a small twist angle with the hexagonal boron nitride $(\mathrm{hBN})$ substrate ${ }^{15}$.

Here, we provide direct evidence for the existence of flat bands in TBG near the magic angle. This is achieved by the combination of low-energy electron microscopy (LEEM) and scanning tunnelling microscopy (STM) with nano-ARPES, a technique that can image the photocurrent with submicron spatial resolution and provide simultaneous and fully independent momentum-space resolution.

Multiple TBG devices were fabricated by the tear-and-stack method (see Supplementary Information, section A). The two graphene monolayers are supported by an hBN flake that isolates the structure from a graphite electrode (Fig. 1a). The latter is connected to a prepatterned $\mathrm{Au}$ contact on an $\mathrm{Si} / \mathrm{SiO}_{2}$ substrate. A graphite stripe is used to connect the TBG to the second electrode. Both the TBG and graphite bottom electrode were grounded for all experiments.

The key difference to TBG devices used for transport experiments is the absence of an hBN flake that encapsulates the structure. This allows unimpeded access for surface techniques but poses a challenge for device fabrication. In particular, it has not been possible to make such open TBG devices with a twist angle homogeneity that rivals that of encapsulated devices ${ }^{3,19}$. In addition, the

\footnotetext{
'Department of Quantum Matter Physics, University of Geneva, Geneva, Switzerland. ${ }^{2}$ Institut de Ciencies Fotoniques, The Barcelona Institute of Science and Technology, Castelldefels, Spain. ${ }^{3}$ Huygens-Kamerlingh Onnes Laboratory, Leiden Institute of Physics, Leiden University, Leiden, The Netherlands. ${ }^{4}$ Elettra-Sincrotrone Trieste S.C.p.A., Basovizza, Trieste, Italy. ${ }^{5}$ Kavli Institute of Nanoscience, Delft University of Technology, Delft, The Netherlands. ${ }^{6}$ National Institute for Materials Science, Tsukuba, Japan. ${ }^{7}$ Department of Theoretical Physics, University of Geneva, Geneva, Switzerland. ${ }^{8}$ Swiss Light Source, Paul Scherrer Institute, Villigen, Switzerland. ${ }^{9}$ These authors contributed equally: Simone Lisi, Xiaobo Lu, Tjerk Benschop, Tobias A. de Jong. 凶e-mail: felix.baumberger@unige.ch
} 
a

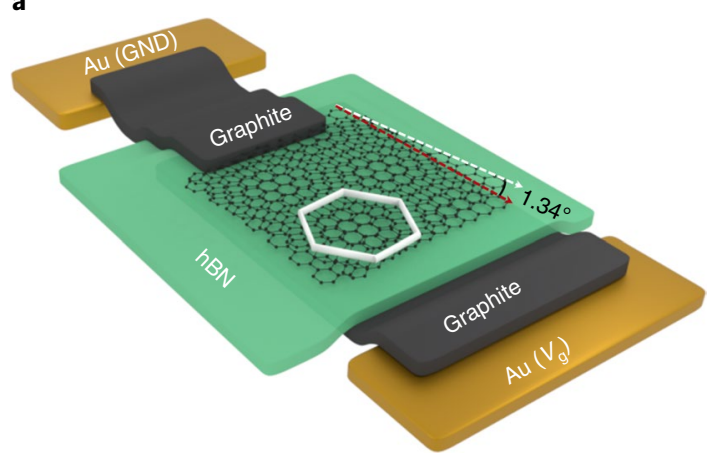

b

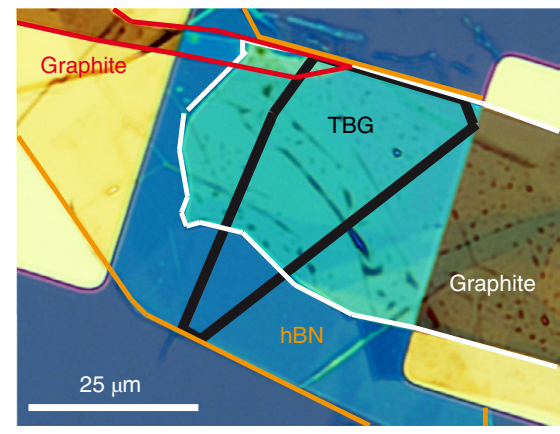

Fig. 1 | Device layout. a, Sketch of the van der Waals stack with TBG on top of hBN and a bottom graphite electrode. The TBG is contacted by a graphite stripe. Note that the twist angle of $1.34^{\circ}$ of the actual device is exaggerated for graphical clarity. This results in a smaller moire unit cell in the schematic (white hexagon) than in the actual device. b. Optical micrograph of the device with boundaries of the different layers outlined in different colours as guides to the eye. Details of the device fabrication are given in the Methods and Supplementary Information, section A.

actual twist angle of such devices has not been determined from gate-dependent transport experiments. This is a serious obstacle for nano-ARPES experiments, as the twist angle of devices frequently changes during fabrication and therefore cannot be predicted reliably. Finally, the electronic properties of open devices are more susceptible to the degrading effect of polymer residues and hydrocarbon contamination of the surface. Therefore, a thorough characterization of the twist angle and cleanliness of the devices prior to ARPES experiments is essential.

This is achieved here by the combination of LEEM and STM. We use bright-field LEEM for a large-scale characterization of the area in which the two twisted graphene monolayers overlap (Fig. 2b). This shows a large area free of folds and bubbles of gases trapped between the layers, but with several round features with lateral dimensions of typically $2 \mu \mathrm{m}$, which we associate with agglomerates of polymer residues.

Contrast differences between areas are attributed to different local lattice stackings ${ }^{20}$. By combining this information from bright-field LEEM with the dark-field LEEM overview in Fig. 2c, we use this stacking contrast to classify areas. As dark-field imaging shows a strong contrast between $\mathrm{AB}$ and $\mathrm{BA}$ stacked Bernal graphene, we can identify unambiguously the large, homogeneous, intermediate intensity area in Fig. $2 \mathrm{c}$ as TBG, which corresponds to a slightly lower intensity in Fig. 2b. It is separated by small folds, visible as dark straight lines, from areas that have reconstructed into Bernal stacking. Some of these areas exhibit alternating AB and BA stacked triangles and can be identified as TBG with a very small twist angle ${ }^{21}$. The smallest of such structures that we can resolve have a line pitch of $25 \mathrm{~nm}$, which corresponds to a twist angle of $0.55^{\circ}$. As no such structures can be observed in the homogeneous TBG area, the moiré period must be smaller there; that is, below the

Fig. 2 | Device characterization. a, Intensity map of the ARPES signal integrated over $3.3 \mathrm{eV}$ from the chemical potential along a $k$-space cut that crosses the K-point of one of the graphene layers in TBG. b, Bright-field LEEM image at a landing energy of $0.2 \mathrm{eV}$ of the area indicated in $\mathbf{a}$. Boundaries between different stacking contrasts are indicated with dashed lines as guides to the eye. c, Dark-field image at a landing energy of $28.0 \mathrm{eV}$ for the area indicated by the white box in $\mathbf{b}$. Triangular reconstruction of low twist angle is visible on the right. d, STM topography acquired with a set-up voltage and current of $V=-250 \mathrm{mV}$ and $I=100 \mathrm{pA}$, respectively. Inset, Fourier transform of a larger area used for the determination of the twist angle, as described in Methods. $q_{0}$ is a reciprocal lattice vector of the moiré superlattice. e, ARPES measurements at the positions indicated with red dots in $\mathbf{c}$, which show a high level of homogeneity in the sample.

resolution of these measurements. This implies that the twist angle in the homogeneous TBG area is larger than $0.55^{\circ}$. Furthermore, in microscopic low-energy electron diffraction, no moiré satellite

a

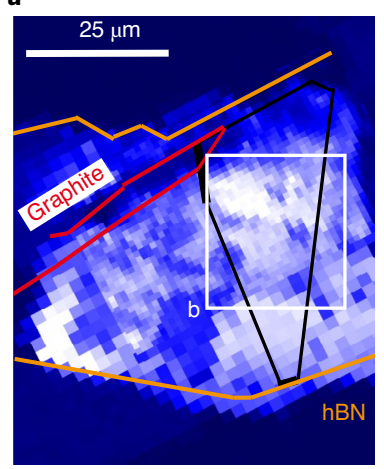

b

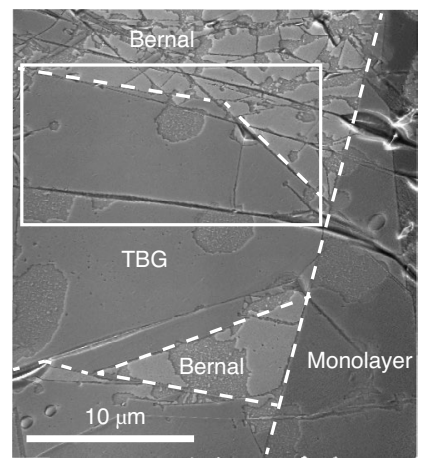

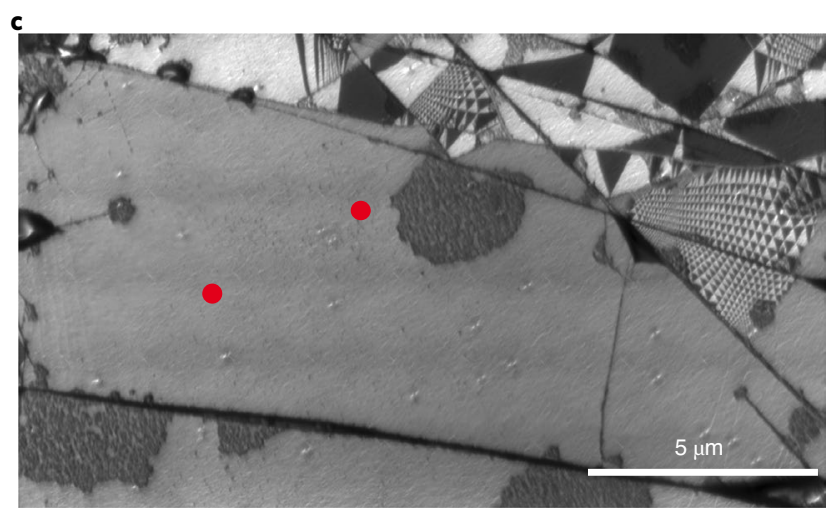

d

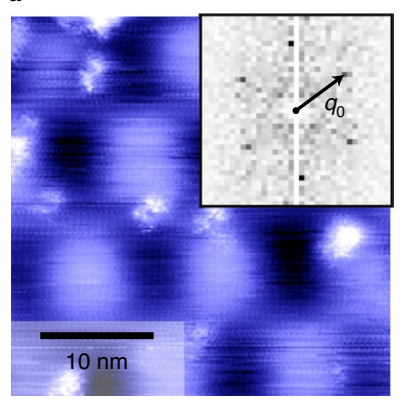

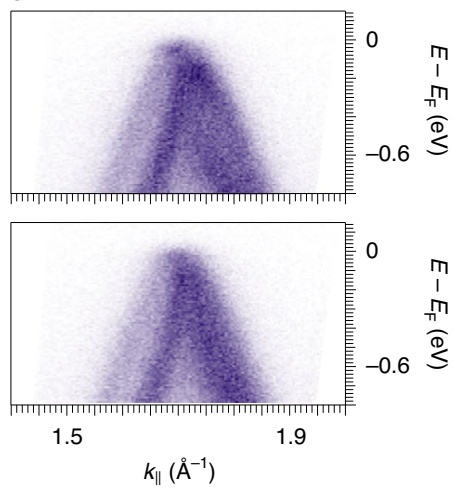


a

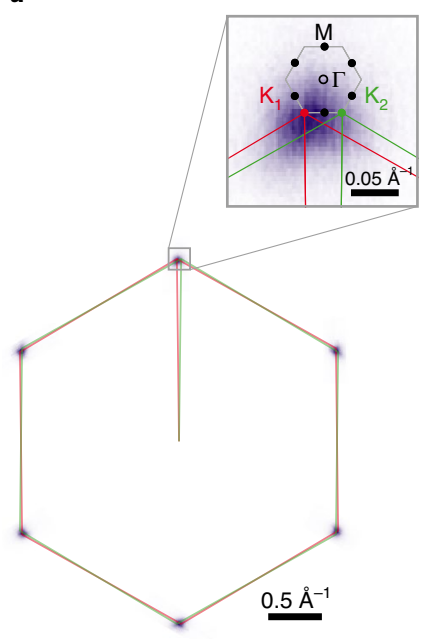

b
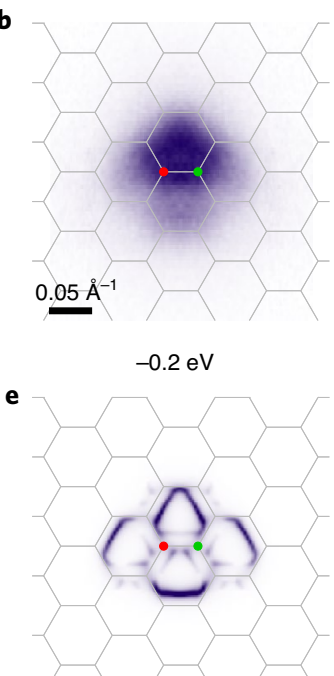

c

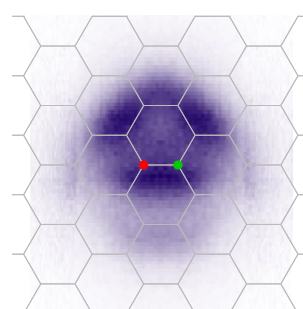

$-0.4 \mathrm{eV}$

f

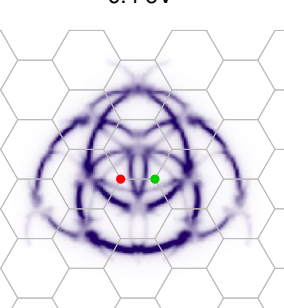

d
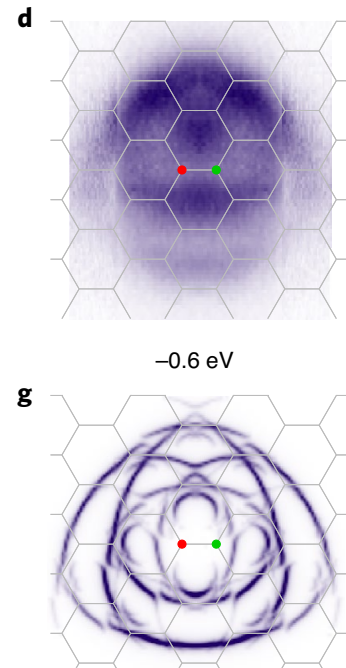

Fig. 3 | ARPES spectral weight distribution. a, Spectral weight at the Fermi level over the full Brillouin zones of the two graphene monolayers (red, green). Inset, a zoomed-in view of the area near the $\mathrm{K}_{1,2}$ points of the two layers (marked by red and green points throughout this figure). The grey hexagon shows the moiré mini Brillouin zone with the high-symmetry $\Gamma$ and $M$ points. b-d, ARPES constant energy contours at $-0.2 \mathrm{eV},-0.4$ eV and $-0.6 \mathrm{eV}$. The data have been symmetrized along the vertical axis. The original unsymmetrized data are shown in Supplementary Fig. 2 . The hexagonal tiles are moiré mini-Brillouin zones. $\mathbf{e}-\mathbf{g}$, Calculations of the spectral weight within the continuum model. A Lorentzian broadening of 10 meV has been applied to the calculation. For details of the calculations, see the Supplementary Information.

peaks are observed for the TBG area, which indicates that the separation between the peaks there is smaller than the resolution that is achieved in these experiments. From this, we obtain an upper bound of $2^{\circ}$ for the twist angle. The same microscopic low-energy electron diffraction patterns confirm an angle of $29 \pm 1^{\circ}$ between TBG and hBN. We have deliberately chosen such a large angle to reveal the intrinsic electronic structure of TBG by the minimization of competing moiré effects from the interaction with hBN.

For a more precise determination of the twist angle in bilayer graphene, we use STM topographic images of the moiré superlattice acquired on the same device (Fig. 2d). From Fourier transforms of large images (Supplementary Fig. 3), we find periodicities of $10.3 \mathrm{~nm}$ to $10.8 \mathrm{~nm}$, which correspond to a variation of the twist angles between $1.31^{\circ}$ and $1.37^{\circ}$ over a distance of approximately $1 \mu \mathrm{m}$ that is probed by these experiments. Further evidence for a good level of homogeneity of the TBG areas comes from position-dependent nano-ARPES experiments. Two representative dispersion plots acquired at different positions on the same TBG area are shown in Fig. 2e and show excellent reproducibility. None of the main features change noticeably between these two spots. This is a prerequisite for the reliable acquisition of the detailed three-dimensional ARPES data sets, which we discuss below.

The application of the concept of band structures is not straightforward for TBG. TBG is translationally invariant and therefore a crystal that supports Bloch states in a strict sense ${ }^{8,22}$ for only a discrete set of twist angles. In the general incommensurate structure, the spectrum of eigenvalues is dense at every momentum, which is fundamentally different from the continuous $E(k)$ dispersion relation that is typical of electrons in simple crystals. Yet, experiments on TBG show clear evidence for band-like transport at any twist angle, irrespective of whether the structure is commensurate or not $^{2,3,16}$. This can be understood by supposing the formation of a quasi band structure from the non-uniform distribution of spectral weights over the complex eigenvalue spectrum, as was proposed for incommensurate density wave systems ${ }^{23}$. ARPES directly measures these spectral weights ${ }^{23,24}$. Indeed, we find that the photoemission intensity at the Fermi level is highly localized near the $K_{1}$ and $K_{2}$ points of the two twisted monolayers from where it disperses away with increasing energy (Fig. 3a). The spectral weight thus singles out a small subset of all possible low-energy eigenvalues. This provides direct support for the emergence of Bloch-like bands out of the complex spectrum of eigenvalues in a moire structure, and thus for the applicability of the widely used continuum models of the band structure.

The effect of the twist angle on the details of the band structure is profound. In Fig. 3, we show a series of constant energy cuts and compare them to band structure calculations of the spectral weight for an isolated TBG layer with a twist angle of $1.34^{\circ}$. As our focus here is on the identification of the salient features of the TBG band structure rather than on a quantitative comparison of different theoretical approaches, we perform all calculations in the widely used continuum model of freestanding TBG with parameters from the literature (see Supplementary Information, section D). Therefore, fully quantitative agreement with the data is not expected. At all energies, we find a far more complex electronic structure than in Bernal bilayer graphene, in which constant energy contours are simple concentric circles with small trigonal warping. The electronic structure observed in this experiment is also fundamentally different from that of bilayer graphene with a large twist angle in which constant energy contours are well described by two weakly hybridized circles centred at $\mathrm{K}_{1}$ and $\mathrm{K}_{2}\left(\right.$ ref. ${ }^{25}$ ). Instead, on TBG we find a complex spectral weight texture with multiple contours that appear to be centred at the $\Gamma$ points of the four mini-Brillouin zones that surround $\mathrm{K}_{1,2}$. This is particularly evident in the calculation at $-0.2 \mathrm{eV}$, but similar features can be recognized at higher energy levels, as well as in the data. We interpret these $\Gamma$-centred constant energy contours as the result of hybridization of Dirac cones at all K-points of the moiré mini-Brillouin zone. This naturally results in band extrema at $\Gamma$ rather than at $\mathrm{K}_{1,2}$, as observed for large twist angles. Our observation of such structures therefore provides direct evidence for the strong interlayer coupling and the formation of moiré mini-bands that is predicted for small-angle $\mathrm{TBG}^{6-8,22}$. We note that some Fermi surfaces appear to be broken into arc-like structures that seemingly end at arbitrary momenta. However, these structures probably do not represent genuine Fermi arcs, such as those observed in Weyl semimetals ${ }^{26}$ and possibly in 


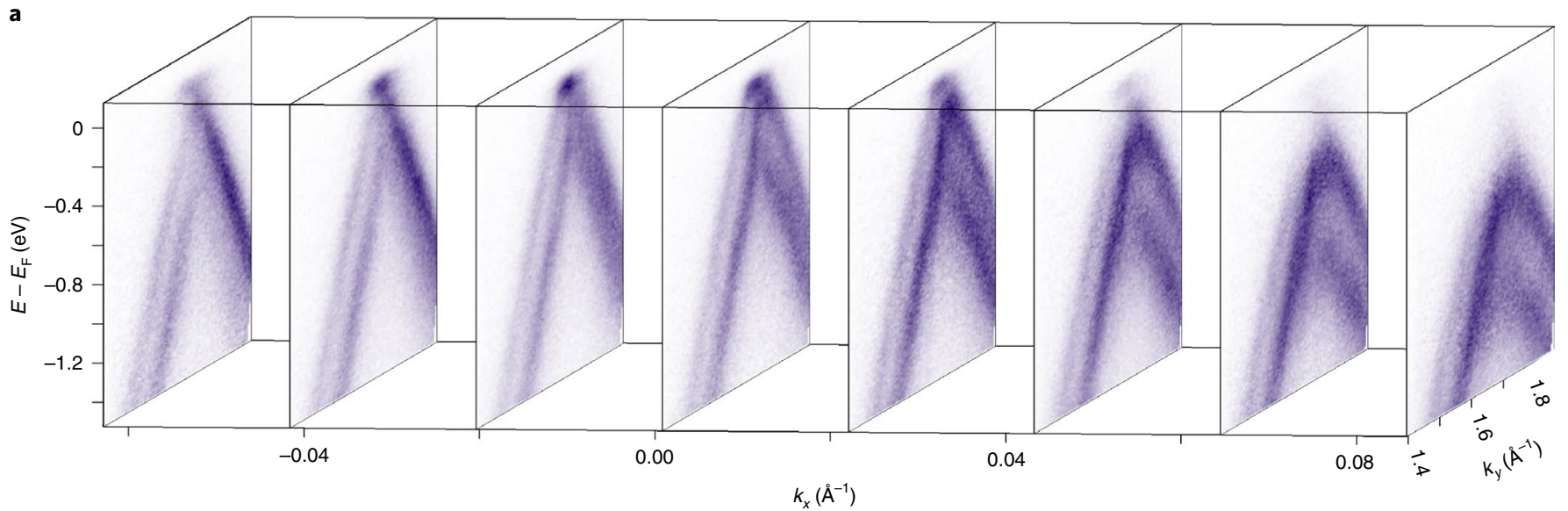

b

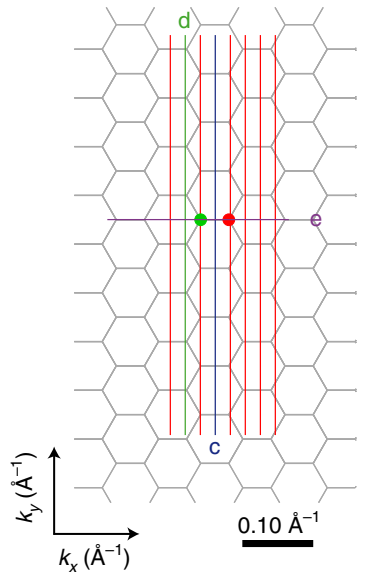

c
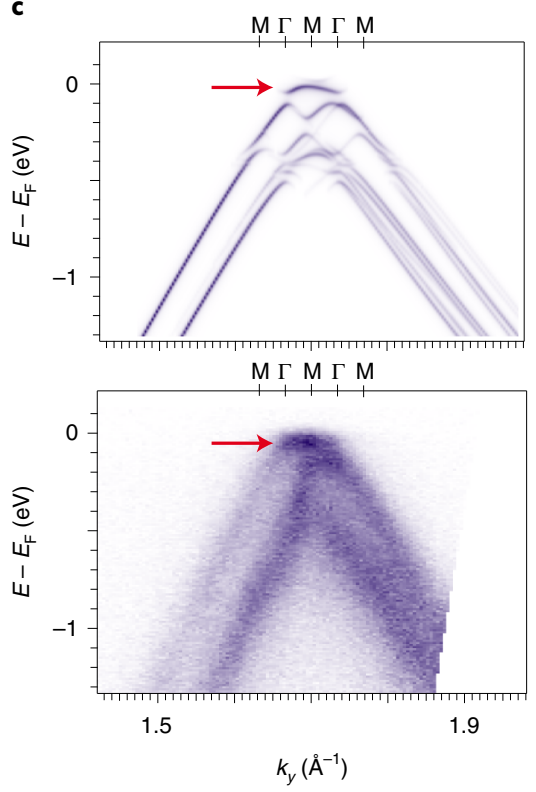

d
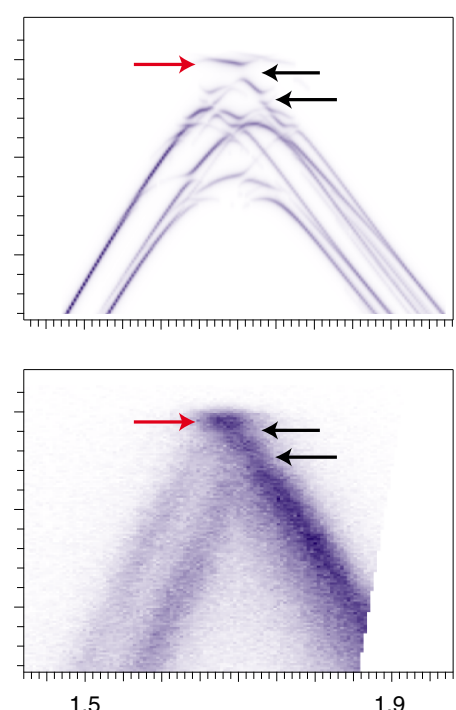

$k_{y}\left(\AA^{-1}\right)$

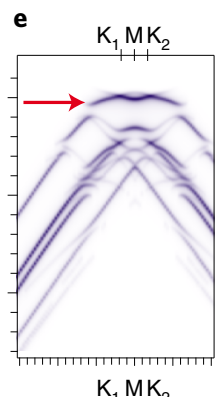

$\mathrm{K}_{1} \mathrm{MK}_{2}$

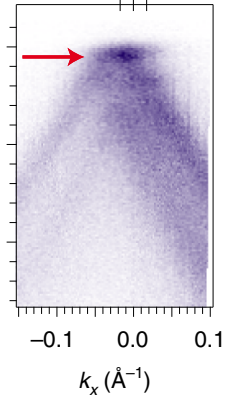

Fig. 4 | Flat band and hybridization gaps. a, Dispersion plots taken on the grid of vertical lines indicated in $\mathbf{b}$. Green and red dots in $\mathbf{b}$ mark the $K_{1,2}$ points of the twisted graphene layers. Lines c, d, and e (highlighted in different colors) mark the momentum space cuts probed in panels $\mathbf{c}$, $\mathbf{d}$ and $\mathbf{e}$, respectively. c-e, Comparison of individual cuts (bottom panels) with calculations of the spectral weight distribution (top panels). The latter use a Lorentzian broadening of $10 \mathrm{meV}$. The flat band and multiple hybridization gaps are marked by red and black arrows, respectively. The high-symmetry points $\Gamma, \mathrm{M}$ and $\mathrm{K}_{1,2}$ refer to the mini-Brillouin zone and are defined in Fig. 3a.

cuprates $^{27}$. Instead, a detailed inspection of the calculations suggests that they emerge from a multitude of very small but closed surfaces whose spectral weight decays away from $\mathrm{K}_{1,2}$ but remains finite (see Supplementary Fig. 3).

We now focus on the $E(k)$ dispersion relation of the quasi-Bloch bands of TBG. The overview of cuts perpendicular to the $\mathrm{K}_{1}-\mathrm{K}_{2}$ line (Fig. 4a) already reveals a dichotomy of the electronic states with two distinct subsystems, as predicted. Most importantly, the raw data directly show a flat band with a spectral weight localized near the $\mathrm{K}_{1,2}$ points that is separated from the dispersive bands. The dispersive bands can, at first approximation, be attributed to the $\mathrm{K}_{1}$ and $\mathrm{K}_{2}$ Dirac cones split by approximately $4 u^{\prime}$ along this $k$-space direction, where $u^{\prime}$ is the interlayer coupling (see Supplementary Information, section D). The detailed comparison of selected cuts with calculations of the spectral weight distribution reveals additional features (Fig. 4c-e). First, we find clear evidence for hybridization gaps in the dispersive bands that reflect the moiré superlattice potential (Fig. 4d). The dominant gaps have magnitudes of up to approximately $100 \mathrm{meV}$ and are therefore comparable to $u^{\prime}$. We note that the calculations further predict a multitude of smaller gaps that arise from a confluence of both hybridization and higher-order Umklapps. These cannot be resolved in our data, which have an energy resolution of $45 \pm 5 \mathrm{meV}$ (see Methods).

The spectral weight at the Fermi level corresponds to a flat band, as shown in Fig. 4c-e. The localization of its spectral weight in $k$-space reflects directly the extended nature of the wave functions in real space ${ }^{22}$. It is difficult to quantify the width of the flat band from fits to our experimental data because fitting results are model dependent, especially with regard to the treatment of the dispersive states, sample imperfections and resolution. From the scatter of different fits, we estimate a $30 \pm 15 \mathrm{meV}$ band width, in fair agreement with our calculations that predict a $46 \mathrm{meV}$ occupied band width, but we cannot exclude an even larger systematic error. More precise measurements of the mini-band width for different twist angles might become possible in future nano-ARPES experiments with improved resolution that follow the procedures outlined here. We note that the flat band is clearly separated from the Dirac bands for most of its extension in $k$-space. For certain cuts, however, it appears 
to almost touch the Dirac bands within the resolution of the experiments. From this, we estimate an upper limit of the gap between these two subsystems of approximately $50 \mathrm{meV}$, which is consistent with calculations that incorporate the effect of structural relaxation in the bilayer ${ }^{28-30}$. The presence of such a gap effectively decouples the flat band from the weakly correlated dispersive states, which is essential for the physics of TBG.

\section{Online content}

Any methods, additional references, Nature Research reporting summaries, source data, extended data, supplementary information, acknowledgements, peer review information; details of author contributions and competing interests; and statements of data and code availability are available at https://doi.org/10.1038/ s41567-020-01041-x.

Received: 12 February 2020; Accepted: 17 August 2020; Published online: 28 September 2020

\section{References}

1. Cao, Y. et al. Correlated insulator behaviour at half-filling in magic-angle graphene superlattices. Nature 556, 80-84 (2018).

2. Cao, Y. et al. Unconventional superconductivity in magic-angle graphene superlattices. Nature 556, 43-50 (2018).

3. Lu, X. et al. Superconductors, orbital magnets and correlated states in magic-angle bilayer graphene. Nature 574, 653-657 (2019).

4. Sharpe, A. L. et al. Emergent ferromagnetism near three-quarters filling in twisted bilayer graphene. Science 365, 605-608 (2019).

5. Yankowitz, M. et al. Tuning superconductivity in twisted bilayer graphene. Science 363, 1059-1064 (2019).

6. dos Santos, J. M. B., Peres, N. M. R. \& Castro Neto, A. H. Graphene bilayer with a twist: electronic structure. Phys. Rev. Lett. 99, 256802 (2007).

7. Suárez Morell, E., Correa, J. D., Vargas, P., Pacheco, M. \& Barticevic, Z. Flat bands in slightly twisted bilayer graphene: tight-binding calculations. Phys. Rev. B 82, 121407 (2010).

8. Bistritzer, R. \& MacDonald, A. H. Moiré bands in twisted double-layer graphene. Proc. Natl Acad. Sci. USA 108, 12233 LP-12237 (2011).

9. Kerelsky, A. et al. Maximized electron interactions at the magic angle in twisted bilayer graphene. Nature 572, 95-100 (2019).

10. Xie, Y. et al. Spectroscopic signatures of many-body correlations in magic-angle twisted bilayer graphene. Nature 572, 101-105 (2019).

11. Jiang, Y. et al. Charge order and broken rotational symmetry in magic-angle twisted bilayer graphene. Nature 573, 91-95 (2019).
12. Li, G. et al. Observation of Van Hove singularities in twisted graphene layers. Nat. Phys. 6, 109-113 (2010).

13. Choi, Y. et al. Electronic correlations in twisted bilayer graphene near the magic angle. Nat. Phys. 15, 1174-1180 (2019).

14. Tomarken, S. L. et al. Electronic compressibility of magic-angle graphene superlattices. Phys. Rev. Lett. 123, 046601 (2019).

15. Utama, M. I. B. et al. Visualization of the flat electronic band in twisted bilayer graphene near the magic angle twist. Nat. Phys. https://doi. org/10.1038/s41567-020-0974-x (2020).

16. Polshyn, H. et al. Large linear-in-temperature resistivity in twisted bilayer graphene. Nat. Phys. 15, 1011-1016 (2019).

17. Razado-Colambo, I. et al. NanoARPES of twisted bilayer graphene on SiC: absence of velocity renormalization for small angles. Sci. Rep. 6, 27261 (2016).

18. Marchenko, D. et al. Extremely flat band in bilayer graphene. Sci. Adv. 4, eaau0059 (2018).

19. Uri, A. et al. Mapping the twist-angle disorder and Landau levels in magic-angle graphene. Nature 581, 47-52 (2020).

20. Hibino, H., Wang, S., Orofeo, C. M. \& Kageshima, H. Growth and low-energy electron microscopy characterizations of graphene and hexagonal boron nitride. Prog. Cryst. Growth Charact. Mater. 62, 155-176 (2016).

21. Yoo, H. et al. Atomic and electronic reconstruction at the van der Waals interface in twisted bilayer graphene. Nat. Mater. 18, 448-453 (2019).

22. Koshino, M. et al. Maximally localized Wannier orbitals and the extended Hubbard model for twisted bilayer. Phys. Rev. X 8, 031087 (2018).

23. Voit, J. et al. Electronic structure of solids with competing periodic potentials. Science 290, 501-503 (2000).

24. Amorim, B. General theoretical description of angle-resolved photoemission spectroscopy of van der Waals structures. Phys. Rev. B 97, 165414 (2018).

25. Peng, H. H. et al. Substrate doping effect and unusually large angle Van Hove singularity evolution in twisted bi- and multilayer graphene. Adv. Mater. 29, 1606741 (2017).

26. Armitage, N. P., Mele, E. J. \& Vishwanath, A. Weyl and Dirac semimetals in three dimensional solids. Rev. Mod. Phys. 90, 015001 (2017).

27. Shen, K. M. et al. Nodal quasiparticles and antinodal charge ordering in $\mathrm{Ca}_{2-x} \mathrm{Na}_{x} \mathrm{CuO}_{2} \mathrm{Cl}_{2}$. Science 307, 901-904 (2005).

28. Nam, N. N. T. \& Koshino, M. Lattice relaxation and energy band modulation in twisted bilayer graphene. Phys. Rev. B 96, 075311 (2017).

29. Gargiulo, F. \& Yazyev, O. V. Structural and electronic transformation in low-angle twisted bilayer graphene. 2D Mater. 5, 015019 (2017).

30. Lucignano, P., Alfè, D., Cataudella, V., Ninno, D. \& Cantele, G. Crucial role of atomic corrugation on the flat bands and energy gaps of twisted bilayer graphene at the magic angle $\sim 1.08^{\circ}$. Phys. Rev. B 99, 195419 (2019).

Publisher's note Springer Nature remains neutral with regard to jurisdictional claims in published maps and institutional affiliations.

(C) The Author(s), under exclusive licence to Springer Nature Limited 2020 


\section{Methods}

Fabrication. A schematic of the fabrication processes is shown in Supplementary Fig. 1. First, hBN flakes were exfoliated onto a PDMS stamp. Then two graphene flakes on a $\mathrm{SiO}_{2} / \mathrm{Si}$ substrate were picked up sequentially with $\mathrm{hBN}$ on PDMS. The two pieces of graphene came from a single graphene flake that was pre-cut with an atomic force microscopy tip and were manually twisted by $1.3^{\circ}$. To ensure that the $\mathrm{hBN}$ flake picked up graphene instead of dropping down on the $\mathrm{SiO}_{2} / \mathrm{Si}$ substrate, the $\mathrm{hBN}$ flake was always kept in partial contact with the substrate during the pick-up process (Supplementary Fig. 1b,c). Subsequently, the TBG-hBN structure was flipped over and picked up with a second PDMS stamp and then transferred onto a graphite flake that was pre-transferred onto the $\mathrm{SiO}_{2} / \mathrm{Si}$ substrate and connected with an Au electrode as a gate. Finally, a second piece of graphite was placed between the TBG and a second prepatterned Au electrode as a contact.

Prior to the measurements shown here, samples were annealed at $\sim 350^{\circ} \mathrm{C}$ in ultrahigh vacuum for several hours.

Nano-ARPES. Experiments were performed at the SpectroMicroscopy beamline of the Elettra light source ${ }^{31}$. This instrument uses multilayer, coated Schwartzschild objectives with a numerical aperture of 0.2 to de-magnify a pinhole located at an intermediate focus on the sample and achieves a spatial resolution of $\sim 600 \mathrm{~nm}$. All of the experiments were performed at $T=85 \mathrm{~K}$ with a photon energy of $27 \mathrm{eV}$ and $p$-polarized light with a fixed incidence angle of $45^{\circ} . k$-space mappings were performed by the rotation of an imaging hemispherical analyser mounted on a five-axis goniometer (instrument built by Elettra). The combined energy and momentum resolution of the experiments was $\sim 45 \mathrm{meV}, 0.005 \AA^{-1}$.

LEEM. Before photoemission electron microscopy and LEEM imaging, samples were annealed at $350^{\circ} \mathrm{C}$, as measured by a pyrometer. Imaging was performed at the same temperature to prevent beam contamination. Images were recorded in high-dynamic-range mode and corrected for detector artefacts, as described in ref. ${ }^{32}$. Photoemission electron microscopy imaging was performed using an unfiltered mercury short-arc lamp with its main emission at a photon energy of $\sim 6 \mathrm{eV}$. Dark-field imaging was performed under tilted illumination, as described in detail in ref. ${ }^{33}$. Furthermore, overviews were stitched together using a cross-correlation-based method and intensity matched globally.

STM measurements. The devices were inserted in our home-built, low-temperature $(4.2 \mathrm{~K})$, ultrahigh-vacuum $\left(<3.0 \times 10^{-10} \mathrm{mbar}\right)$ set-up, which features a commercial STM head (RHK Technology) and a cryostat (CryoVac). The devices were then annealed to $350^{\circ} \mathrm{C}$ for approximately $10 \mathrm{~h}$ before insertion into the STM head. To land the STM tip on the TBG sample, we used the capacitive navigation method described in ref. ${ }^{34}$. Our $\mathrm{Si} / \mathrm{SiO}_{2}$ chip contained a patterned gold contact, on which we applied an $\mathrm{AC}$ voltage of $V_{\mathrm{pp}}=1 \mathrm{~V}$ at $5 \mathrm{kHz}$ with respect to ground. The same signal, but rotated $180^{\circ}$ out of phase, was applied to the Si chip. We then used the coarse motor to move the tip laterally at a distance of a few micrometres above the sample, and used the strength of the capacitive signal to guide the movement with respect to the gold pattern. Once the TBG flake was located, we approached the tip to perform the STM measurements. All STM measurements were performed with mechanically polished PtIr tips (Unisoku).

Extraction of the twist angle. To determine the twist angle, we Fourier transformed topographic images of $113 \times 113 \mathrm{~nm}^{2}$ and measured the distance $\left|q_{0}\right|$ in $q$ space to each moiré peak (Supplementary Fig. 2). The wavelength $\lambda_{\mathrm{M}}$ of the moiré lattice was then determined by the calculation of the moiré wavelength $\lambda_{\mathrm{M}}=\frac{4 \pi}{\sqrt{3}\left|q_{0}\right|}$. Finally, the twist angle $\theta$ was obtained using the formula $\lambda_{\mathrm{M}}=\frac{a}{2 \sin \frac{\theta_{2}}{2}}$ where $a=0.246 \mathrm{~nm}$ is the graphene lattice constant.

Calculations. To compute the theoretical ARPES intensity, we used a nearest-neighbour tight-binding model to model each layer and the standard twisted continuum theory to model the interlayer coupling. The ARPES intensity was obtained by the projection of the electron wave functions for twist angle $\theta=1.34^{\circ}$ onto the first mini-Brillouin zone (for details, see Supplementary Information, section D).

\section{Data availability}

Supporting data are available for this paper in ref. ${ }^{35}$. All other data that support the plots within this paper and other findings of this study are available from the corresponding author upon reasonable request.

\section{References}

31. Dudin, P. et al. Angle-resolved photoemission spectroscopy and imaging with a submicrometre probe at the SPECTROMICROSCOPY-3.2L beamline of Elettra. J. Synchrotron Radiat. 17, 445-450 (2010).

32. de Jong, T. A. et al. Quantitative analysis of spectroscopic low energy electron microscopy data: high-dynamic range imaging, drift correction and cluster analysis. Ultramicroscopy 213, 112913 (2020).

33. de Jong, T. A. et al. Intrinsic stacking domains in graphene on silicon carbide: a pathway for intercalation. Phys. Rev. Mater. 2, 104005 (2018).

34. Li, G., Luican, A. \& Andrei, E. Y. Self-navigation of a scanning tunneling microscope tip toward a micron-sized graphene sample. Rev. Sci. Instrum. 82, 073701 (2011)

35. Lisi, S. et al. Source data for 'Observation of flat bands in twisted bilayer graphene'. Available at https://doi.org/10.26037/yareta:nxtqgcnllzbklcak4qyzi3 adv4 (2020).

\section{Acknowledgements}

We thank J. Aarts, S. Nadj-Perge, A. Yazdani, A. Pasupathy, A. Morpurgo, I. Gutierrez-Lezama, H. Henck, F. Groenewoud, K. van Oosten, R.M. Tromp, R. Wijgman and H. Zandvliet for discussions. We thank M. Hesselberth for technical LEEM support. The ARPES work was supported by the Swiss National Science Foundation (SNSF) through grant 200020_184998. L.R. acknowledges support by the SNSF through an Ambizione grant. The STM work was supported by the European Research Council (ERC StG SpinMelt) and by the Dutch Research Council (NWO), as part of the Frontiers of Nanoscience programme, as well as through a Vidi grant (680-47-536). The LEEM work was supported by the NWO as part of the Frontiers of Nanoscience programme. Growth of hBN crystals was supported by the MEXT Element Strategy Initiative to Form Core Research Center (JPMXP0112101001) and the Core Research for Evolutional Science and Technology (JPMJCR15F3), Japan Science and Technology Agency. D.K.E. acknowledges support from the Ministry of Economy and Competitiveness of Spain through the Severo Ochoa programme for Centres of Excellence in R\&D (SE5-0522), Funda-ció Privada Cellex, Fundació Privada Mir-Puig, the Generalitat de Catalunya through the CERCA programme, the H2020 Programme (820378), 2D-SIPC and the La Caixa Foundation.

\section{Author contributions}

X.L., P.S. and J.R.D. made the TBG devices. T.T. and K.W. contributed hBN materials. S.L., F.M., I.C., E.C. and A.H. performed the nano-ARPES experiments. T.B., V.S. and M.L. performed the STM experiments. T.A.d.J. acquired the LEEM and microscopic low-energy electron diffraction data. L.R. performed the band structure calculations. J.J., S.J.v.d.M. (LEEM), M.A. (STM), D.K.E. (devices) and F.B. (nano-ARPES) were responsible for the project supervision and the provision of resources. V.K., A.G. and A.B. were responsible for the nano-ARPES beamline. S.L., A.T. and F.B. wrote the bulk of the manuscript with contributions from several others. All authors contributed to the scientific discussion of the results.

\section{Competing interests}

The authors declare no competing interests.

\section{Additional information}

Supplementary information Supplementary information is available for this paper at https://doi.org/10.1038/s41567-020-01041-x.

Correspondence and requests for materials should be addressed to F.B.

Peer review information Nature Physics thanks Zhongkai Liu and the other, anonymous, reviewer(s) for their contribution to the peer review of this work.

Reprints and permissions information is available at www.nature.com/reprints. 